\title{
Fine-Grained Frequencies Meet Synchronous Transmission
}

This paper was downloaded from TechRxiv (https://www.techrxiv.org).

\section{LICENSE}

CC BY-NC-SA 4.0

SUBMISSION DATE / POSTED DATE

$16-11-2021 / 29-11-2021$

CITATION

Debadarshini, Jagnyashini; Saha, Sudipta (2021): Fine-Grained Frequencies Meet Synchronous Transmission. TechRxiv. Preprint. https://doi.org/10.36227/techrxiv.17021849.v2

$\mathrm{DOI}$

10.36227/techrxiv.17021849.v2 


\title{
Fine-Grained Frequencies Meet Synchronous Transmission
}

\author{
Jagnyashini Debadarshini, IIT Bhubaneswar \\ Sudipta Saha, IIT Bhubaneswar
}

\begin{abstract}
Fine-grained frequencies have been used in several recent works to enhance network throughput as well as combat Cross Technology Interference (CTI) issues in licence free ISM bands. We observe that synchronous communication based strategies, due to the scope of inter-frequency capture-effect, are inherently more capable of supporting in-parallel communication over multiple channels even when the Center Frequency Distance (CFD) of the channels are very low ( $<5 \mathrm{MHz}$ ). In this work, we pursue an in-depth study of how fine-grained frequencies can be used in conjunction with synchronous communication to extract the maximum benefit from a very narrow band of available frequencies (e.g., 2 - $5 \mathrm{MHz}$ ) for in-parallel intra-group communication. In this direction, we propose a simple and efficient group formation strategy to automatically define groups in a given WSN/loT network to boost up in-parallel intra-group communication efficiency. Through extensive experimental evaluations in existing WSN/loT testbeds, we show that in-parallel one-to-all dissemination in the groups formed through the proposed strategy can execute with upto $73 \%$ higher reliability while consuming upto $41 \%$ lower energy as compared to the same running among the groups formed through naive strategy with only 4 consecutive frequencies separated by $1 \mathrm{MHz}$ and upto 20 groups.
\end{abstract}

Index Terms-WSN, loT, Concurrent transmission, Capture-effect, Continuous frequency allocation, Overlapping channels.

\section{INTRODUCTION}

A smart-system such as, smart-home, smart-agriculture, smart-city [1] etc., primarily thrive upon a decentralized interaction among a large number of smart-devices. Technologies like Internet-of-Things (IoT)/Wireless Sensor Networks (WSN) serve a major role in these smart-systems. Dense deployment of the devices is quite common in these systems, e.g., in a smart-factory a large number of static/mobile robots/robotic-arms or in a smart-agriculture a large number of drones would require to work together. The communication protocols used in these smart-systems often suffer from scalability issues [2], [3] which may create a huge bottleneck in any such large and dense decentralized system. Dividing the set of devices into groups and inparallel execution of different fragment of the target job by different groups have been a promising solution approach in the existing works [4], [5], [6]. Such simultaneous intragroup communication activities are best supported by Frequency Division Multiplexing (FDMA).

For in-parallel communication and throughput enhancement, FDMA has been applied in development of MAC protocols in WSN/IoT, e.g., McMAC [7], [8], MMSN [9], TMCP [10] to name a few. However, in all these multichannel MAC protocols the existence of a sufficient number of good channels is essential for their successful operation. IoT/WSN systems use the licence free ISM bands for communication. Due to an exponential rise in the number of devices operating in the ISM bands as well as various communication technologies (e.g., BLE, ZigBee, LoRa, WiFi, Microwave oven etc.) sharing the same band, it results in severe CTI issues. It degrades the performance of any multi-channel MAC protocol because of unavailability of sufficient number of channels/frequencies or instability of the available channels requiring frequent change and researching of channels.

Use of fine-grained frequencies in the ISM band, has been one of the strategies to solve the said issue [11], [12]. Under $2.4 \mathrm{GHz}$ ISM band there are total 16 ZigBee channels separated by a CFD of $5 \mathrm{MHz}$. With a CFD of 1 $\mathrm{MHz}$, the same band contains 80 fine-grained frequencies (2405 MHz to $2484 \mathrm{MHz}$ ). However, due to low CFD, interfrequency interference becomes a significant issue while using these fine-grained frequencies for in-parallel communication. Different solutions have been proposed to mitigate this problem. For example, the work FAVOR [11] proposes a spatial tessellation technique which takes into account the inter-node/link distance for appropriate assignment of the fine-grained frequencies. The work [12] uses dynamic CCA threshold adjustment and apply a minimum of $3 \mathrm{MHz}$ separation to achieve fruitful solution.

In summary, the existing works on fine-grained frequencies depend on availability of some detailed physical information such as inter-node or inter-group distance etc. These requirements impose a significant restriction in the applicability of these strategies in general. The existing works use CSMA/CA for their MAC operation. These works have also indirectly shown that transmitting packets concurrently/synchronously can exploit the fine-grain frequencies in a better way. But the studies are done in a fully controlled setting and also do not reveal a clear understanding of the said fact. Synchronous transmission based strategies are gaining quite a good attention in many recent works [13], [14], [15]. However, as far as the authors' knowledge goes, there has been no study so far on the dynamics of the use of fine-grained frequencies in conjunction with the standard concurrent transmission based protocols. In this work we take an endeavor to carry out a systematic study of this 
important issue.

In a nutshell, under synchronous transmission, two different packets are allowed to be transmitted at the same time. Such concurrent transmissions, in spite of colliding with each other, under certain constraints, succeed even without any channel separation. It becomes possible by the virtue of the phenomena called capture-effect [13], [14]. This fact motivates us to study the capture-effect in interfrequency context. Especially, we focus on understanding how an addition of a slight frequency separation can benefit capture-effect or relax the associated constraints of captureeffect. The work [6] reports our initial study in this direction. In the current work, we begin with an in-depth study of the fundamental characteristics of the communication operations under low-CFD based separation in synchronous transmission. Next, we propose a strategy to automate the process of extracting the group setting which can provide the best outcome for network-wide in-parallel intra-group activities. The main contributions of the work are summarized below.

- Detailed study of the effectiveness of very low-CFD based separation (from $1 \mathrm{MHz}$ ) under synchronous transmission with real IoT devices.

- Exhaustive identification of the possible scenarios and exploration of the requirements to achieve higher efficiency under low-CFD inter-group separation.

- A decentralized automated group formation strategy to form the set of desired number of groups for efficient intra-group in-parallel communication.

- The full implementation of group formation strategy in Contiki OS for TelosB devices. Extensive comparison with a naive group formation strategy in publicly available testbeds for IoT/WSN.

The rest of the paper is organized as follows. Section 2 provides a brief description of the existing multi-channel as well as fine-grained frequency based works in WSN/IoT. Section 3 summarizes the fundamental concepts, the necessary background and the overall design framework used in our work. Section 4 depicts our study based on the dynamics of synchronous communication under low-CFD based separation in a controlled local setting. Section 5 provides the design of the proposed group formation strategy. Section 6 reports the experiment based evaluation study of the proposed strategy in publicly available WSN/IoT testbeds. Section 7 concludes the work with some future directions.

\section{Related works}

Use of multiple channels in WSN has been a common target in many prior works [16], [17], [18], [19], [20], [21]. These works assume availability of enough good and orthogonal channels which is very difficult to achieve in reality due to CTI and congestion in the ISM bands. Works such as [22], [23] address this problem by dynamic channel switching. However, a significant overhead is incurred in such switching. Thus, these solutions work well only when a good many CTI free channels are available.

Continuous non-orthogonal frequencies are used in works such as [11], [12], [24]. They bring better utilization of the spectrum and solve the channel scarcity problem upto a certain extent. However, these works exploit physical information for frequency assignment to the nodes for inparallel activities. For instance, the work [11], depends on the physical distance between the links. It assigns arbitrary frequencies to groups/links that are far from each other and different frequencies to groups/links close to each other. But obtaining physical information is a great bottleneck in many situations. In contrast, we avoid use of any physical information. Rather, we automate the process of extracting the cluster of nodes which can do in-parallel communication under very limited frequency resource.

The works [11], [12] point out that the traditional CSMA/CA mechanism reduces the possibility of in-parallel activities because of CCA. In [24], as an extension of [11], the authors propose switching-off CSMA/CA in a probabilistic manner to improve throughput and the packet reception rate of the network. On the contrary, the work [12] combats the problem through a dynamic CCA adjustment (DCA) method. It first shows that inter-channel interference has significantly lesser impact than the co-channel interference. Based on this, it identifies the range of the signal strength for the co-channel and inter-channel interference. Next, it sets the CCA threshold in a way so that concurrent transmissions in neighboring channels are allowed while avoiding co-channel packet collisions as much as possible.

The existing works directly or indirectly indicate that synchronous/concurrent transmission have great potential to make better use of the fine-grained frequencies compared to asynchronous transmission. But there has not been any systematic study so far on this issue. Under synchronous transmission the MAC operations are fully redefined. Unlike traditional CSMA/CA based protocols, in synchronous communication, by the virtue of special physical layer phenomena capture-effect, transmissions are allowed at the same time even in the same channel/frequency. Thus, intuitively, addition of even a $1 \mathrm{MHz}$ of frequency gap can enhance the scope of capture-effect more in the interfrequency context due to diminished degree of interference. This indicates a great potential in the use of synchronous transmission to exploit consecutive fine-grained frequencies in a more effective way which we explore in our current work.

\section{BACKGROUND AND THE BASIC IDEA}

In $2.4 \mathrm{GHz}$ ISM band, when a pair of nodes communicate using 802.15.4 radio in a certain channel, because of the bandwidth requirement, some components of the signal leak in the adjacent channels. But the strength of the leaked signals decreases very fast with CFD. To avoid problems due to such leakage, communication activities are performed only in the standard channels separated by $5 \mathrm{MHz}$ gap. Although, 802.15.4 compatible radios allow the access to all these $1 \mathrm{MHz}$ separated channels, the effect of interfrequency interference becomes visible as the CFD goes below $5 \mathrm{MHz}$. Hence, in $2.4 \mathrm{GHz}$ band, out of 80 frequencies, only 16 are usable.

In-parallel communication is quite possible in frequencies that are away from each other. However, in presence of CTI and heavy congestion it maybe much easier to find a 
very narrow band of CTI free frequencies [25], [26]. Thus, in order to use the fine-grained frequencies for sustained inparallel activities in such situations, its required to exploit them the best under very low CFD (below $5 \mathrm{MHz}$ ) which is the target of the current study.

FDMA with fully orthogonal channels, the communications can happen independently. While, the CFD between channels decreases, the inter-frequency interference increases and ideally is the highest when multiple communications happen in the same frequency. Below, we revise the possible scenarios that can appear when two packet transmissions overlaps in the same frequency.

1) Constructive interference: Based on the rules of physics, when two signals perfectly overlap with each other, and they bear the same content, a stronger signal is created through constructive interference. However, in reality its hard to have perfectly overlapping signals. It has been shown in the work Glossy [13], that under 802.15.4 the transmitters need to remain synchronized at the level of $0.5 \mu \mathrm{S}$ to result in constructive interference.

2) Capture-effect: In practice its hard to maintain synchronization within $0.5 \mu \mathrm{S}$. Thus, constructive interference is difficult to achieve. Many wireless radios can differentiate packets having stronger signal when multiple packets arrive almost at the same time. This is referred to as capture-effect. There are two conditions for capture-effect to happen successfully. First, the strongest signal will have to be at least $3 \mathrm{~dB}$ stronger than the sum of the signalstrength of the other packets. This is called the condition for power-capture. Second, the strongest packet should appear within $160 \mu \mathrm{S}$ (in 802.15.4) from the start of the arrival time of the very first packet. This is called the condition for time-capture. Note that unlike constructive-interference, captureeffect does not need the contents of packets to be same.

3) Collision: This is the usual case which happens when the overlap between the packets do not meet the criteria of neither constructive-interference nor capture-effect.

When multiple packets arrive almost at the same time at an $\mathrm{RF}$ receiver, both the physical layer phenomena constructive-interference and capture-effect provide a scope for the receiver to select one out of these packets. Captureeffect allows this even when the contents of the packets are different. Conversely, through appropriate adjustment of transmission power or node-positioning, by the virtue of capture-effect, a pair of nodes can exchange data even when another pair of nodes are communicating in the same channel/frequency. Thus, capture-effect inherently supports in-parallel communication. However, from the perspective of interference, capture-effect faces the maximum challenge when it takes places in the same frequency. At a CFD of even $1 \mathrm{MHz}$, due to a slight frequency separation, the situation theoretically goes more in favor of capture-effect because of the reduction in the strength of the leaked signal in the adjacent frequency. The fundamental goal of this work is to understand how well we can exploit inter-frequency capture-effect to facilitate simultaneous communication activities.

Synchronous transmission: Collision among multiple packets diminishes the efficiency of any protocol. To avoid collision, protocols following asynchronous mode of communication, e.g., Aloha or CSMA/CA based MACs, try to avoid the overlap in the transmissions of the packets. Overlap among multiple packets, in these protocols, mostly happens in an arbitrary way as shown in Figure 1. Possibility of capture-effect happening naturally from such overlaps is very rare and thus leads to collision. Thus, the benefit of capture-effect cannot be achieved much through asynchronous strategies. On the contrary, synchronous transmissions try to avoid collision by letting the packets overlaps perfectly which lead to either constructive interference or capture effect (see Figure 1). Thus, synchronous communication inherently promotes capture effect which motivates us to use it in our work.

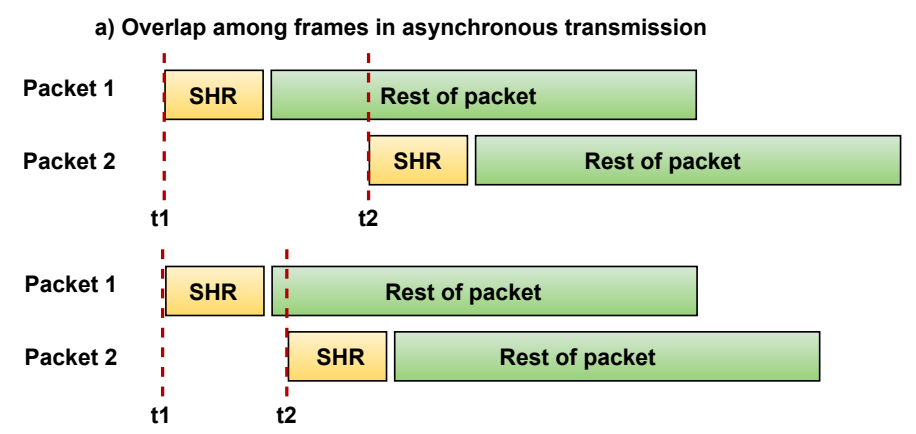

b) Overlap among frames in synchronous transmission

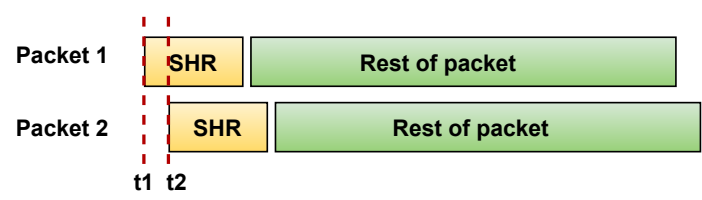

Fig. 1. Overlap among frames when packet arrives in synchronous and asynchronous communication.

Overall Framework: In order to exploit synchronous transmission, we borrow the concept of software defined networking (SDN) for WSN/IoT introduced in the work Atomic-SDN [27]. A Glossy [13] based flood is used for network-wide time-synchronization and dissemination of information regarding the type of SDN service. Followed by this, the actual network operations take place. We use a similar SDN based framework for in-parallel intra-group dissemination. In summary, we assume that a given network is divided into a number of groups (of nodes) and each group carries out communication (one-to-all dissemination) activities in a synchronous manner.

The overall setting is summarized below. A round of Glossy denoted as Outer-Glossy, is used for a quick networkwide time-synchronization as well as dissemination of control information. Subsequently, the phase denoted by GrpGlossy is used for dividing the network into multiple groups. Simultaneous intra-group communication takes place in the next phase Inner-Glossy. Grp-Glossy is discussed in details in Section 5. We experiment with six fine-grained FDMA strategies referred by $G_{0}, G_{1}, G_{2}, G_{3}, G_{4}$, and $G_{5}$, where $G_{i}$ 
implies that the groups are assigned frequencies with a gap of $i \mathrm{MHz}$.

Groups can be defined either manually or through automated strategy in the Grp-Glossy phase. The framework with three manually defined groups is pictorially depicted in Figure 2. Left side (a) shows the groups with defined initiator nodes (I1 for Group-1, I2 for Group-2, and I3 for Group-3). The right side (b) shows the processes running in some of the sampled nodes from each of the groups (N1 from Group-1, N2 and N3 from Group-2, and N4 from Group-3) through a time-diagram. Since, the groups are defined manually, the Grp-Glossy phase does not have any function although shown in the diagram. The OuterGlossy runs network-wide while the Inner-Glossy runs in each group independently and simultaneously in a way so that all intra- and inter-group operations happen in a timealigned manner as much as possible. To maintain the same, we also fix a moderate packet-size globally, although packet contents can be different in different group. Appropriate padding is used to meet the requirement. Outer-Glossy disseminates the control information, e.g., packet-size, group membership. With manual group definition, it also shares the frequency for each node/group to be used in InnerGlossy execution while with automated group formation, the Outer-Glossy shares only the frequency-range to be used. During the actual group formation process in GrpGlossy, as described in Section 5, every group initiator independently sets a random frequency from that range for its group. Thus, the whole system runs in a decentralized fashion. After a round of intra-group dissemination in the Inner-Glossy, all the nodes tunes back to a default frequency to participate in the Outer-Glossy.

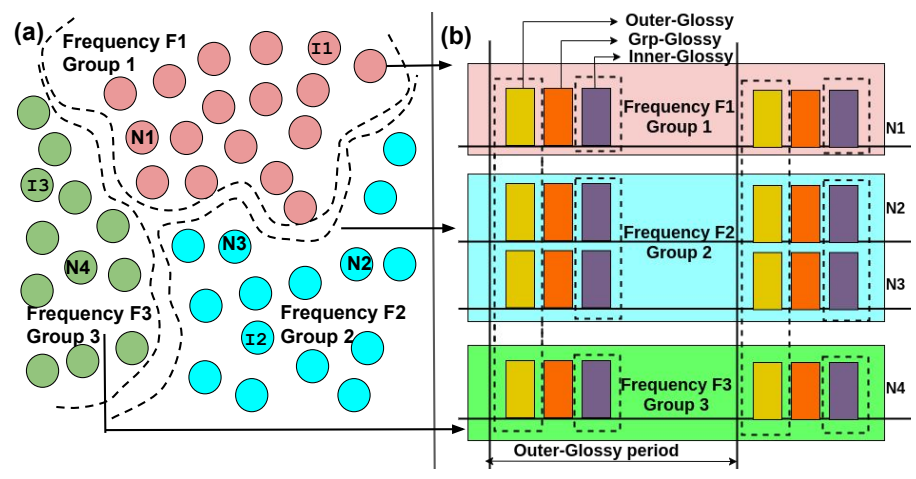

Fig. 2. Overall framework of the proposed simultaneous intra-group oneto-all dissemination strategy.

\section{STUDY BASED ON CONTROLLED LOCAL EXPER- IMENTS}

Conjunction of synchronous transmission and fine-grained frequencies works best in a setting that can favor interfrequency capture-effect. When two packets arrive at a receiver approximately at the same time, whether the setting is going in favor of capture-effect or not, depends on various possible situations. To get a decent idea, we first exhaustively list out all these situations based on the constraints for time-capture and power-capture [28], [29].
Based on the time of arrival of the inter- and intragroup packets at the receiver, seven independent situations are possible as detailed below.

1) Case-t1: Both the packets arrive almost at the same time.

2) Case-t2: Inter-group packet arrives approximately $64 \mu \mathrm{S}$ later favoring the intra-group packet little-bit. But this does not violate the capture-effect constraint from the perspective of inter-group packet.

3) Case-t3: Inter-group packet arrival is delayed for approx. $256 \mu S$, thus violating time-constraint for capture effect from the point of view of inter-group packet while favoring intra-group packet little more.

4) Case-t4: Inter-group packet arrival is delayed for $t_{\delta}$ $m s$, where $t_{\delta} \geq$ intra-group packet transmission time. It leads to non-overlapping inter- and intra-group transmissions.

5) Case-t5: Intra-group packet arrives about $64 \mu \mathrm{S}$ after inter-group packet making the situation favorable for inter-group packet.

6) Case-t6: Intra-group packet arrival is delayed for about $256 \mu S$ which violates time-capture constraint.

7) Case-t7: Intra-group packet arrival is delayed for $t_{\delta} \mathrm{ms}$, where $t_{\delta} \geq$ inter-group packet transmission time, i.e., there is no overlap in the packet transmissions.

Similarly, based on power-capture constraints, four possible scenarios can be there for each of the seven cases mentioned above. These are detailed below.

1) Case-p1: Both the packets bear almost same signal strength.

2) Case-p2: Intra-group packet bears approximately $3 d B$ - $4 d B$ larger signal strength. Naturally, this goes in favor with intra-group packet.

3) Case-p3: Inter-group packet bears approximately $3 d B$ $-4 d B$ larger signal strength. This goes in favor with inter-group packet.

4) Case-p4: Inter-group packet bears $6 \mathrm{~dB}$ to $8 \mathrm{~dB}$ larger strength than intra-group creating a very hostile situation.

In summary, under controlled time-capture, in Case-t 1 the two packets arrive at the same time while in Case-t4 and Case- $t 7$ the packets appear in a non-overlapping way. Among the rest, Case- $t 2$ and Case- $t 3$ favor intra-group packet while Case- $t 5$ and Case-t 6 favor the inter-group packet. Similarly, under controlled power-capture, Case- $p 1$ provides equal benefit to both the packets while Case-p2 and Case-p3 favor the intra- and inter-group packet, respectively. Lastly, Case- $p 4$ shows a more hostile situation by favoring intergroup packet more. To gain a decent idea on how the above-mentioned cases conflict with or favor capture-effect, we carry out controlled experiments in a local setting as described below.

Experimental setup: A miniature version of the framework described in the previous section is derived where three TelosB devices (MoteA, MoteB and MoteC), are used to form two groups. MoteA and MoteB are assumed to belong to the same group (group-1) where MoteB acts as the transmitter (initiator) and MoteA acts as the receiver. MoteC is assumed to be in different group (group-2) and 
acts as the interfere. The packet from the same group, i.e., from MoteB to MoteA is referred to as the intra-group packet while the packet from other group, i.e., from MoteC as the inter-group packet. The motes are placed over a straight-line at equal distances (one meter) as shown in Figure 3. OuterGlossy phase runs in the beginning of every iteration. There is no Grp-glossy as the group information is hard coded in this setting. Two groups are assigned with two different frequencies as per the strategy $\left(G_{i}\right)$ being tested. Under $G_{0}$ a single frequency is assigned to both the groups. In InnerGlossy, both MoteB and MoteC transmit one packet of size 56 bytes having different randomly generated data. Hence, the value of $t_{\delta}$, in the emulation of the Case-t4 and Case$t 7$, is set to $1.8 \mathrm{~ms}$. To fruitfully carry-out an experiment on power-capture in $G_{i}$, we adjust the transmission power levels of MoteB and MoteC by running the same experiment first over $G_{0}$ and observing the RSSI values in the received packets.

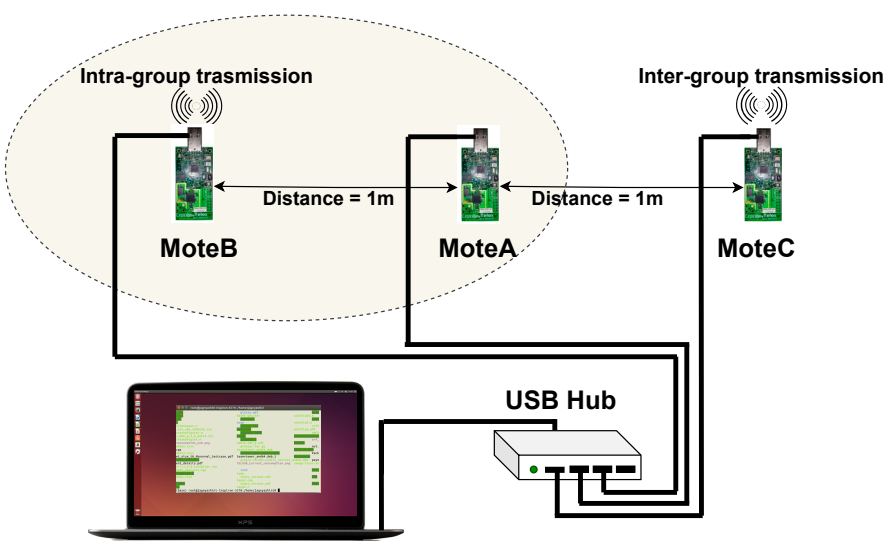

Fig. 3. Local experimental setup with three motes forming two groups.

Metrics: Each experiment is repeated for 1000 iterations. In each iteration MoteA can receive a packet from MoteB either successfully or in a corrupted form due to simultaneous transmission from the interfere MoteC. The later one is referred to as collision. Moreover, in case a packet is successfully received by MoteA, it maybe a packet from the interfere (in case of $G_{0}$ only) or a legitimate packet from MoteB. The former is referred to as intrusion while the later one as reception. In each experiment we measure these three quantities. The reception and intrusion are presented in percentage for all the $7 \times 4$, i.e., 28 cases in Table 1 . The collision percentage can be calculated as the three quantities (intrusion, collision and reception) sum up to 100 .

\subsection{Results}

From the results as shown in Table 1, it can be observe that $G_{3}$ and above gap show $100 \%$ reception in all the 28 cases which goes in line with the findings so far available in the literature. The experiments reveal the merits of all the low-CFD separations including $G_{0} . G_{0}$ performs very well in about $29 \%$ of these cases. $G_{1}$, and $G_{2}$, have been seen to work extremely well in $46 \%$ and $82 \%$ of all the possible cases, respectively. Its revealed that $G_{1}$ succeeds even when only power capture is satisfied and time capture is not satisfied by the intra-group packet. Using $G_{0}$ in such cases leads to packet corruption as the reception of intergroup packet starts earlier while high power intra-group packet arrives later. Similar behavior has been observed in $G_{2}$ also. Intra-group packets are favored by $G_{2}$ when both the intra- and inter-group packets have almost equal strength or even inter-group packets have a $3 \mathrm{~dB}-4 \mathrm{~dB}$ higher strength than the intra-group packet irrespective of time-capture. However, when the strength of inter-group packets increase to more than $6 \mathrm{~dB}$ as compared to the intragroup packets, due to stronger inter-group packets the intragroup packets start getting corrupted.

Note that all the cases mentioned above are studied independently under controlled setting. But in fact, chance of occurrence of these cases are vastly different from each other and depend on the specific setting used. In general under asynchronous transmission, there can be either avoidance of packet overlaps through MAC protocols such as CSMA/CA, or arbitrary overlaps among packets which do not meet the constraint of time-capture. Use of synchronous transmission in our approach automatically enables us to get control on the time-capture. Thus, although $G_{0}, G_{1}$ and $G_{2}$ are found to be performing good in lesser number of cases compared to $G_{3}$ and others, the chances of these cases under synchronous transmission are quite high which is the primary reason behind their overall success [6].

However, maximum benefit from the fine-grained frequencies can be obtained when the constraints for both time- and power-capture are met. But constraints to achieve power-capture are harder to meet. Dynamic CCA adjustment [12], distance based frequency assignment [11], [30] are some of the approaches toward that direction only. We approach in a novel way through appropriate formation of the clusters/groups to support power-capture naturally as detailed in the next section.

\section{DESIGN}

Constraints of power-capture can be met by controlling the transmission power so that intrusion by the inter-group packets are restricted. However, its hard to decide which node need to control transmission power in what way in a dynamic or decentralized scenario. In contrast, the formation of the groups also can be done in a way that can inherently meet the power capture requirements through appropriately selecting the nodes and defining the intergroup border accordingly. However, as the number of nodes in the network as well as groups increase, it becomes hard to set the groups even in a manual way to satisfy such constraints.

Fundamentally the power-capture constraints are best satisfied when there is good inter-group separation. The work [6] uses a metric called Disjointedness $(D)$ to capture this inter-group separation. $D$ for a node is defined as the ratio of the number of its neighbors that belong to same groups and total number of its neighbors. $D$ for a network for a certain group division is calculated as the the sum of the said ratios over all the nodes in the network. Through a variety of manually defined groups the work [6] shows that success in simultaneous intra-group dissemination is proportional to $D$. However, obtaining a group division in a network satisfying a certain value of $D$ 


\begin{tabular}{|c|c|c|c|c|c|c|c|c|c|}
\hline \multirow{2}{*}{$\begin{array}{l}\text { SL } \\
\text { No }\end{array}$} & \multirow{2}{*}{$\begin{array}{l}\text { Packet arrival time } \\
\text { (Time-capture) }\end{array}$} & \multirow{2}{*}{$\begin{array}{c}\text { Difference in signal } \\
\text { strength (Power-capture) }\end{array}$} & \multirow{2}{*}{ Status } & \multicolumn{6}{|c|}{ Frequency Gap } \\
\hline & & & & G0 & G1 & G2 & G3 & G4 & G5 \\
\hline \multirow{2}{*}{1} & \multirow{8}{*}{$\begin{array}{l}\text { At the same time } \\
\text { (Case-t1) }\end{array}$} & \multirow{2}{*}{$\begin{array}{l}\text { Almost same signal strength } \\
\text { (Case-p1) }\end{array}$} & Reception & 0 & 0 & 100 & 100 & 100 & 100 \\
\hline & & & Intrusion & 0.92 & 0 & 0 & 0 & 0 & 0 \\
\hline \multirow{2}{*}{2} & & \multirow{2}{*}{$\begin{array}{l}\text { Intra-group packet bears 3dB - 4dB larger strength } \\
\text { (Case-p2) }\end{array}$} & Reception & 98.72 & 100 & 100 & 100 & 100 & 100 \\
\hline & & & Intrusion & 0 & 0 & 0 & 0 & 0 & 0 \\
\hline \multirow{2}{*}{3} & & \multirow{2}{*}{$\begin{array}{l}\text { Inter-group packet bears 3dB - 4dB larger strength } \\
\text { (Case-p3) }\end{array}$} & Reception & 0 & 0 & 100 & 100 & 100 & 100 \\
\hline & & & Intrusion & 97.67 & 0 & 0 & 0 & 0 & 0 \\
\hline \multirow{2}{*}{4} & & \multirow{2}{*}{$\begin{array}{l}\text { Inter-group packet bears } 6 \mathrm{~dB}-8 \mathrm{~dB} \text { larger strength } \\
\text { (Case-p4) }\end{array}$} & Reception & 0 & 1.73 & 24.09 & 100 & 100 & 100 \\
\hline & & & Intrusion & 100 & 0 & 0 & 0 & 0 & 0 \\
\hline \multirow{2}{*}{5} & & Almost same signal strength & Reception & 0 & 0.53 & 100 & 100 & 100 & 100 \\
\hline & & (Case-p1) & Intrusion & 1.51 & 0 & 0 & 0 & 0 & 0 \\
\hline & & Intra-group packet bears $3 \mathrm{~dB}-4 \mathrm{~dB}$ larger strength & Reception & 100 & 100 & 100 & 100 & 100 & 100 \\
\hline 6 & Inter-group packet arrives about & (Case-p2) & Intrusion & 0 & 0 & 0 & 0 & 0 & 0 \\
\hline 7 & (Case-t2) & Inter-group packet bears $3 \mathrm{~dB}-4 \mathrm{~dB}$ larger strength & Reception & 0 & 0 & 100 & 100 & 100 & 100 \\
\hline$t$ & & (Case-p3) & Intrusion & 91.06 & 0 & 0 & 0 & 0 & 0 \\
\hline & & Inter-group packet bears $6 \mathrm{~dB}-8 \mathrm{~dB}$ larger strength & Reception & 0 & 3.61 & 17.05 & 100 & 100 & 100 \\
\hline 8 & & (Case-p4) & Intrusion & 100 & 0 & 0 & 0 & 0 & 0 \\
\hline & & Almost same signal strength & Reception & 0.72 & 0 & 100 & 100 & 100 & 100 \\
\hline 9 & & (Case-p1) & Intrusion & 0 & 0 & 0 & 0 & 0 & 0 \\
\hline & & Intra-group packet bears $3 \mathrm{~dB}-4 \mathrm{~dB}$ larger strength & Reception & 100 & 100 & 100 & 100 & 100 & 100 \\
\hline 10 & Inter-group packet arrives about & (Case-p2) & Intrusion & 0 & 0 & 0 & 0 & 0 & 0 \\
\hline 11 & $\begin{array}{c}256 \mu \text { s later } \\
\text { (Case-t3) }\end{array}$ & Inter-group packet bears $3 \mathrm{~dB}-4 \mathrm{~dB}$ larger strength & Reception & 0 & 0 & 100 & 100 & 100 & 100 \\
\hline $1 T$ & & (Case-p3) & Intrusion & 0 & 0 & 0 & 0 & 0 & 0 \\
\hline 12 & & Inter-group packet bears $6 \mathrm{~dB}-8 \mathrm{~dB}$ larger strength & Reception & 0 & 0 & 39.38 & 100 & 100 & 100 \\
\hline 12 & & (Case-p4) & Intrusion & 0 & 0 & 0 & 0 & 0 & 0 \\
\hline & & Almost same signal strength & Reception & 100 & 100 & 100 & 100 & 100 & 100 \\
\hline 13 & & (Case-p1) & Intrusion & 0 & 0 & 0 & 0 & 0 & 0 \\
\hline 14 & & Intra-group packet bears $3 \mathrm{~dB}-4 \mathrm{~dB}$ larger strength & Reception & 100 & 100 & 100 & 100 & 100 & 100 \\
\hline 14 & $\begin{array}{l}\text { Inter-group packet arrives about } \\
18 \mathrm{~ms} \text { later }\end{array}$ & (Case-p2) & \begin{tabular}{|l|} 
Intrusion \\
\end{tabular} & 0 & 0 & 0 & 0 & 0 & 0 \\
\hline 15 & $\begin{array}{c}1.8 \mathrm{~ms} \text { later } \\
\text { (Case-t4) }\end{array}$ & Inter-group packet bears $3 \mathrm{~dB}-4 \mathrm{~dB}$ larger strength & Reception & 100 & 100 & 100 & 100 & 100 & 100 \\
\hline 15 & & (Case-p3) & Intrusion & 0 & 0 & 0 & 0 & 0 & 0 \\
\hline 16 & & Inter-group packet bears $6 \mathrm{~dB}-8 \mathrm{~dB}$ larger strength & Reception & 100 & 100 & 100 & 100 & 100 & 100 \\
\hline 10 & & (Case-p4) & Intrusion & 0 & 0 & 0 & 0 & 0 & 0 \\
\hline 17 & & Almost same signal strength & Reception & 0 & 0 & 100 & 100 & 100 & 100 \\
\hline 17 & & (Case-p1) & \begin{tabular}{|l|} 
Intrusion \\
\end{tabular} & 2.56 & 0 & 0 & 0 & 0 & 0 \\
\hline 18 & & Intra-group packet bears $3 \mathrm{~dB}-4 \mathrm{~dB}$ larger strength & Reception & 100 & 100 & 100 & 100 & 100 & 100 \\
\hline 10 & Intra-group packet arrives about & (Case-p2) & Intrusion & 0 & 0 & 0 & 0 & 0 & 0 \\
\hline 19 & $\begin{array}{r}64 \mu \text { s later } \\
\text { (Case-t5) }\end{array}$ & Inter-group packet bears $3 \mathrm{~dB}-4 \mathrm{~dB}$ larger strength & Reception & 0 & 0 & 100 & 100 & 100 & 100 \\
\hline 19 & & (Case-p3) & Intrusion & 96.75 & 0 & 0 & 0 & 0 & 0 \\
\hline 20 & & Inter-group packet bears $6 \mathrm{~dB}-8 \mathrm{~dB}$ larger strength & Reception & 0 & 0.52 & 12.18 & 100 & 100 & 100 \\
\hline$\angle 0$ & & (Case-p4) & Intrusion & 100 & 0 & 0 & 0 & 0 & 0 \\
\hline 21 & & Almost same signal strength & Reception & 0 & 0 & 100 & 100 & 100 & 100 \\
\hline 21 & & (Case-p1) & Intrusion & 3.27 & 0 & 0 & 0 & 0 & 0 \\
\hline 22 & & Intra-group packet bears $3 \mathrm{~dB}-4 \mathrm{~dB}$ larger strength & Reception & 0 & 100 & 100 & 100 & 100 & 100 \\
\hline $2 \angle$ & Intra-group packet arrives about & (Case-p2) & Intrusion & 0 & 0 & 0 & 0 & 0 & 0 \\
\hline & $\begin{array}{l}256 \mu s \text { later } \\
\text { (Case-t6) }\end{array}$ & Inter-group packet bears $3 \mathrm{~dB}-4 \mathrm{~dB}$ larger strength & Reception & 0 & 0 & 100 & 100 & 100 & 100 \\
\hline 23 & & (Case-p3) & Intrusion & 91.67 & 0 & 0 & 0 & 0 & 0 \\
\hline 24 & & Inter-group packet bears $6 \mathrm{~dB}-8 \mathrm{~dB}$ larger strength & Reception & 0 & 0 & 19.71 & 100 & 100 & 100 \\
\hline 24 & & (Case-p4) & Intrusion & 100 & 0 & 0 & 0 & 0 & 0 \\
\hline & & Almost same signal strength & Reception & 0 & 100 & 100 & 100 & 100 & 100 \\
\hline 25 & & (Case-p1) & Intrusion & 100 & 0 & 0 & 0 & 0 & 0 \\
\hline 26 & & Intra-group packet bears $3 \mathrm{~dB}-4 \mathrm{~dB}$ larger strength & Reception & 0 & 100 & 100 & 100 & 100 & 100 \\
\hline 26 & Intra-group packet arrives about & (Case-p2) & Intrusion & 100 & 0 & 0 & 0 & 0 & 0 \\
\hline 27 & (Case-t7) & Inter-group packet bears $3 \mathrm{~dB}-4 \mathrm{~dB}$ larger strength & Reception & 0 & 100 & 100 & 100 & 100 & 100 \\
\hline 21 & & (Case-p3) & Intrusion & 100 & 0 & 0 & 0 & 0 & 0 \\
\hline 28 & & Inter-group packet bears $6 \mathrm{~dB}-8 \mathrm{~dB}$ larger strength & Reception & 0 & 100 & 100 & 100 & 100 & 100 \\
\hline$\angle 0$ & & (Case-p4) & Intrusion & 100 & 0 & 0 & 0 & 0 & 0 \\
\hline
\end{tabular}

TABLE 1

Summary of each of the 28 cases as detailed in Section 4. 
is hard. Moreover, factors such as initiator-position, intergroup PRR, etc. would also affect power-capture directly or indirectly. Defining groups optimizing all these factors is hard even through manual inspection. In this work we propose a simple group formation mechanism to meet these requirements as detailed below.

Glossy based Automated Group Division Strategy (GDA): We use the concept proposed in the work LEACH [31] under a Glossy based synchronous-communication framework. A set of nodes are selected as the group initiators which start the Glossy flood simultaneously. The nodes in the network gets exposed to multiple floods. However, a non-initiator node accepts a flood and forwards, that it receives first. Thus, in effect, these simultaneous floods compete with each other and incrementally form individual groups. GDA needs to use $G_{0}$. By the virtue of capture-effect the simultaneous floods in GDA become able to make their own space and capture the nodes that are most suitable to form a group. The process stabilizes within a small time and quite successfully decides the inter-group boundaries for a given number of group.

Glossy based Random Group Division Strategy (GDR) In order to compare we carry out the group formation also in offline through a naive way. Through a prior link measurement, a connectivity graph is derived from the given network. The process starts with a defined number of initiators each of which grows an individual group through an iterative process. In each iteration a random neighbor of the existing nodes in each group is selected and added in that group. Once a node is added in a group, it is removed from the pool from where the nodes are selected.

The groups formed by GDA and GDR are compared through the performance of the in-parallel intra-group one-to-all dissemination under various fine-grained FDMA strategies $\left(G_{i}\right)^{1}$.

\section{Evaluation}

The more the intra-group cohesion and the less the intergroup coupling, the more favorable the corresponding group division is to the intra-group power-capture and adverse for inter-group power-capture. GDA tries to naturally form the groups satisfying this basic criteria while GDR provides a set of connected but randomly formed groups. We compare the performance of simultaneous intragroup one-to-all dissemination under GDA and GDR. The experimental settings, parameters, metrics, are described below.

\subsection{Experimental Settings, Parameters, and Metrics}

The following are the main metrics which are used in this evaluation.

Reliability: At node level reliability is defined as the percentage of instances of an experiments where a node receives the information from the respective initiator. We present the average reliability in network level which is the average of the reliability values over all the nodes in the network.

1. To demonstrate the proof of the concept our initial study [6], uses a purely manual strategy to form the groups with an aim to achieve a certain $D$. We do not use it in this study.
Latency: Latency at a node is defined as the amount of time taken to receive a packet from the respective initiator in the dissemination process. We present the average latency which is defined as the average of the latency values of all the nodes in the network.

Radio-on time: It is the total amount of time a node needs to keep its radio on to complete the packet dissemination process. It is presented as the average of the radio-on time values over all the nodes.

N-TX: The intra-group dissemination process is governed by a globally defined parameter N-TX. It is the maximum number of times a node forwards a packet after which it can turn off its radio. N-TX is set based on network size. Higher N-TX implies higher reliability as well as higher radio-on time. In all our experiments we set the value of $\mathrm{N}$ TX to 3.

An experiment is carried out for at least 5000 iterations. The performance metrics are computed on each node separately and averaged over all the iterations and all the nodes. The errorbars in the results represent the standard deviations.

Experimental platforms: Standard simulators such as OMNet++, NS2, NS3, and even in the Cooja simulator that comes with Contiki do not support non-standard frequency assignments. Therefore, we used real devices for all of our experiments. The experiments in all the existing works on fine-grained frequency, are done only on locally developed testbeds consisting of fixed and small groups. In contrast, in the current work we extensively use publicly available WSN/IoT testbeds Indriya [32], FlockLab [33] and DCube [34] comprised of 44, 48 and 25 TelosB motes, respectively.

With increasing congestion in the ISM bands, availability of free frequencies/channel becomes hard. However, it has been reported in prior works that due to spatial correlation, availability of narrow band of consecutive frequencies is more likely and easy to discover than obtaining orthogonal frequencies [25], [26]. Hence, in the current work we consider allocation of frequencies from a band of given size as explained below.

- Frequency band $\left(R_{i}\right)$ : Instead of picking up a frequency in a controlled way as done in [6], in this work we select frequencies from a band of size 1 upto 80 in the $2.4 \mathrm{GHz}$ ISM band. $R_{i}$ denotes a band of $i$ consecutive frequencies. For example, in $R_{1}$ we use a single frequency ( $2480 \mathrm{MHz}$ ) while in $R_{2}$ we use two frequencies, i.e., the band $2479-2480 \mathrm{MHz}$.

- Frequency selection from $R_{i}$ : In each experiment, a group of nodes is assigned with a certain frequency. Determining frequency for a group is itself a nontrivial task. To keep our analysis independent of any frequency allocation strategy, we use the most generic random allocation. In $R_{1}$, since there is only one option, it results in only the strategy $G_{0}$, whereas under $R_{2}$, through random allocation of the frequencies both $G_{0}$ and $G_{1}$ come together, and so on.

\subsection{Results}

Figure 4(a), compares the reliability under GDR (dashed lines) and GDA (solid lines) for different number of target groups (different markers) and frequency band (X-Axis, $R_{i}$ ) 


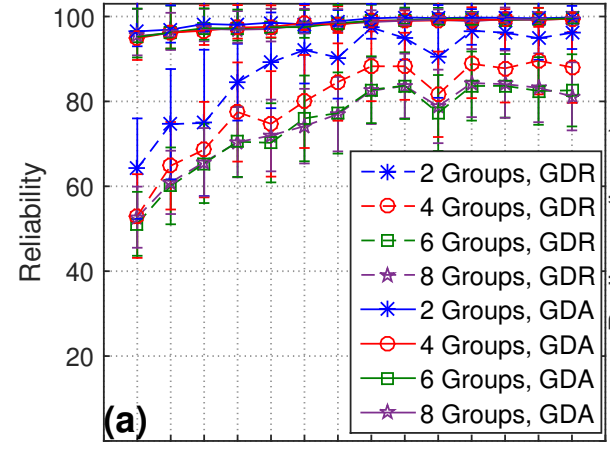

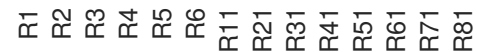
Frequency band

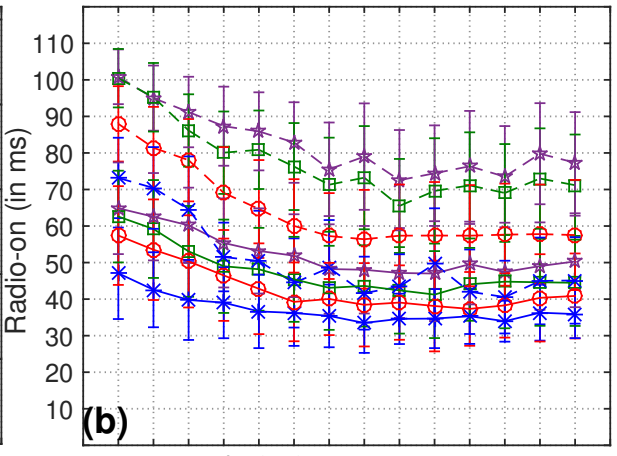

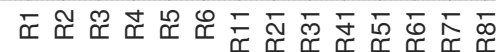
Frequency band

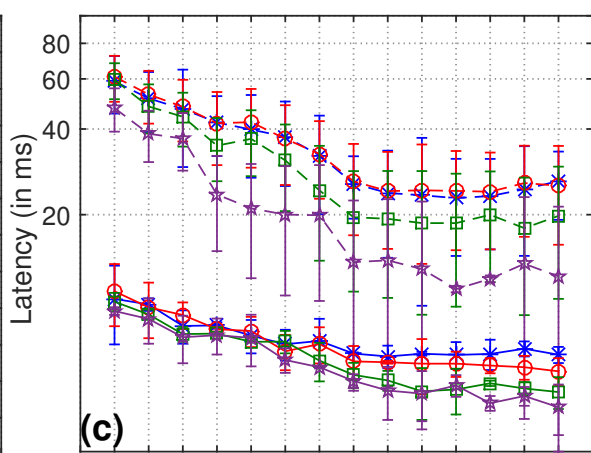

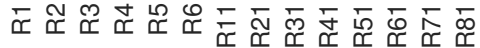
Frequency band

Fig. 4. Effect on reliability (a), radio-on (b) and latency (c) for Flocklab where the groups are formed by GDR and GDA. The y-axis in part (c) is shown in logarithmic scale.

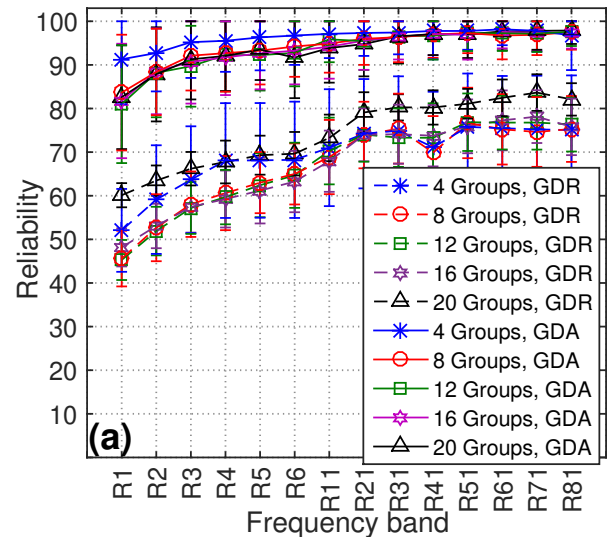

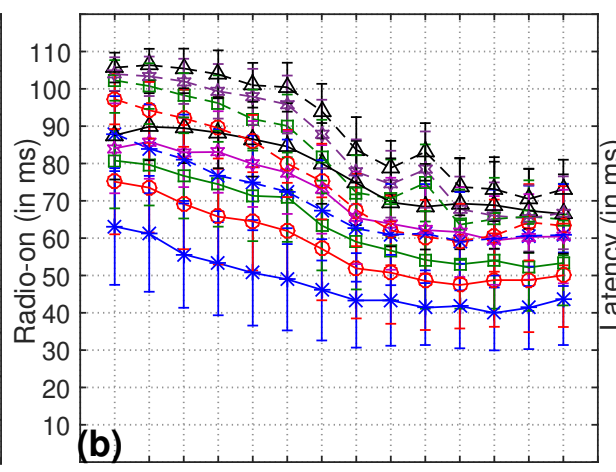

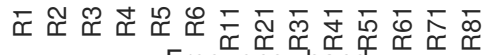

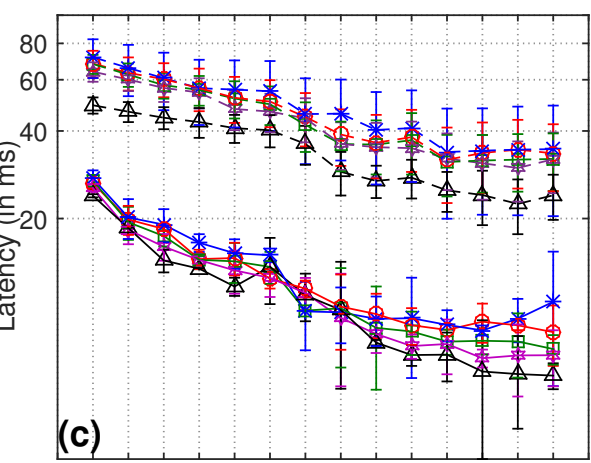

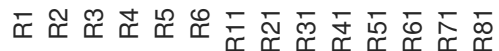
Frequency band

Fig. 5. Effect on reliability (a), radio-on (b) and latency (c) for DCube where the groups are formed by GDR and GDA. The y-axis in part (c) is shown in logarithmic scale.

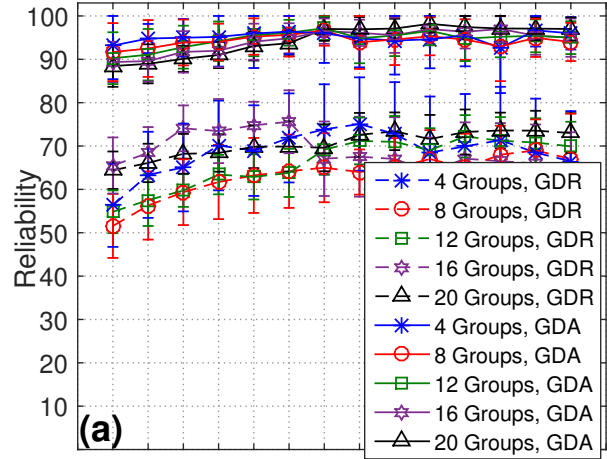

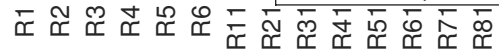
Frequency band

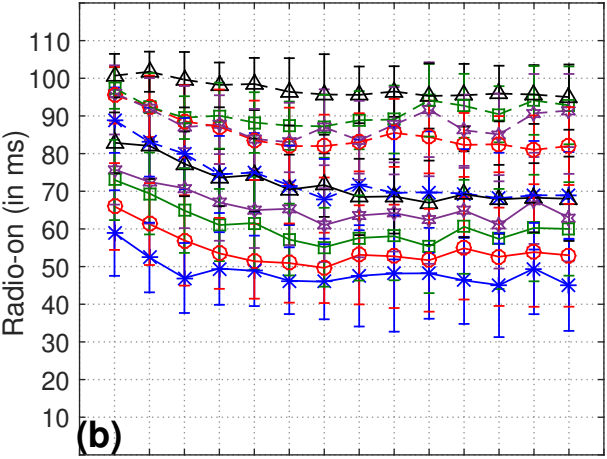

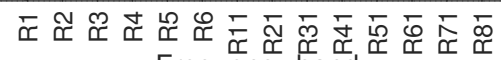
Frequency band

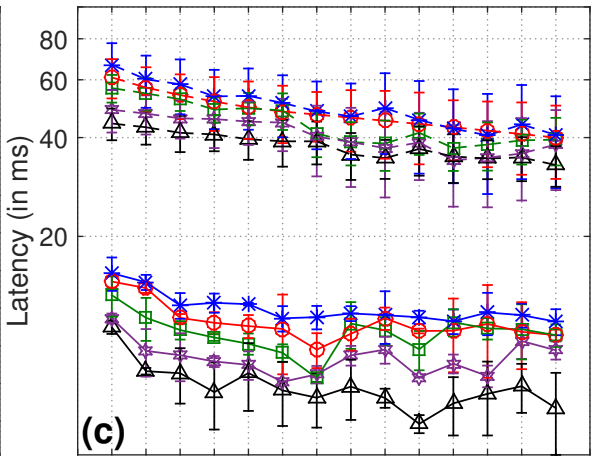

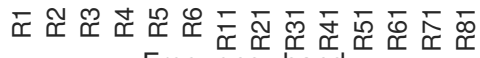
Frequency band

Fig. 6. Effect on reliability (a), radio-on (b) and latency (c) for Indriya where the groups are formed by GDR and GDA. The y-axis in part (c) is shown in logarithmic scale. 
in the $2.4 \mathrm{GHz}$ spectrum in Flocklab. Its visible that with GDA, reliability goes over $90 \%$ even from $R_{1}$ (only $G_{0}$ ) and it goes above $98 \%$ when $G_{0}, G_{1}$ and $G_{2}$ works together, i.e., in $R_{3}$. Reliability remains similar when we allocate more frequencies, i.e., $R_{4}$ and onward. With higher number of groups, situation becomes challenging, because of diminishing group size, most of the nodes get exposed to inter-group interference. However, we observe that for a wide range of group divisions starting from 2 to 8 , where in the worst case every group contains even only three nodes, reliability with GDA remains quite high while it drops significantly (upto $50 \%$ in $R_{1}$ ) under GDR.

Figure 5(a) and 6(a) show the results from DCube and Indriya testbed. Density of the nodes in DCube is quite high while in Indriya its low and almost similar to FlockLab. Due to higher scope of interference in DCube, we see a drop of reliability in $R_{1}$. However, reliability goes quickly up above $98 \%$ as we allocate only four consecutive frequencies (i.e., $R_{4}$ ) even with random allocation. Behavior is almost similar for higher number of groups. In DCube and Indriya we go for even 20 groups where each group contains approximately 2 nodes. Under GDR, dissemination processes gets severely affected by inter-group interference for which the reliability heavily degrades as is visible in the figures. In GDR we donot have any control over the structure of the groups too. In many cases the structure is found to be quite skewed which weakens the cohesion within even a single group. Thus, performance under GDR remains poor even for higher size frequency band.

Figures 4(b) and 4(c), 5(b) and 5(c), and 6(b), and 6(c) show the results of radio-on time and latency in Flocklab, DCube and Indriya, respectively. For a fixed network, higher group count results in lesser group size. Lesser radio-on time and latency are, thus, natural for higher group count as visible in all the results. Significant difference in the radio-on time between GDA and GDR is visible in all the results. The same is also true for latency results. It can be observed that, under higher $R_{i}$, although reliability does not vary much, the process completes much faster in general. Note that the major changes in the metrics are noticeable as it moves from $R_{1}$ to $R_{4}$. For the rest, it remains almost similar.

In a nutshell, in Flocklab, GDA shows an improvement over GDR in reliability upto $73 \%, 60 \%, 48 \%, 37 \%$ and $38 \%$ while consuming upto $40 \%, 39 \%, 38 \%, 36 \%$ and $36 \%$ lesser energy in using 1,2, 3, 4 and 5 consecutive frequencies, respectively. In Indriya, improvements in reliability are $67 \%$, $64 \%, 58 \%, 52 \%$ and $52 \%$ with $41 \%, 40 \%, 38 \%, 36 \%$ and $34 \%$ lesser energy consumption, in using 1, 2, 3, 4 and 5 consecutive frequencies, respectively. In DCube, the improvements in terms of reliability are $70 \%, 69 \%, 58 \%, 54 \%$ and $51 \%$ and in terms of energy consumption are 35\%,33\%,31\%, 30\% and $30 \%$ in using $1,2,3,4$ and 5 consecutive frequencies, respectively.

The results show that GDA can successfully produce groups with high intra-group cohesion and low inter-group coupling which ultimately increases inter-group separation (higher Disjointedness). To correlate we calculate $D$ for each of the groups formed through GDR and GDA. Figure 7 shows the results for each different numbers of groups in FlockLab as a scatter plot of $D$. It can be observed that GDA can successfully divide a network into a de-

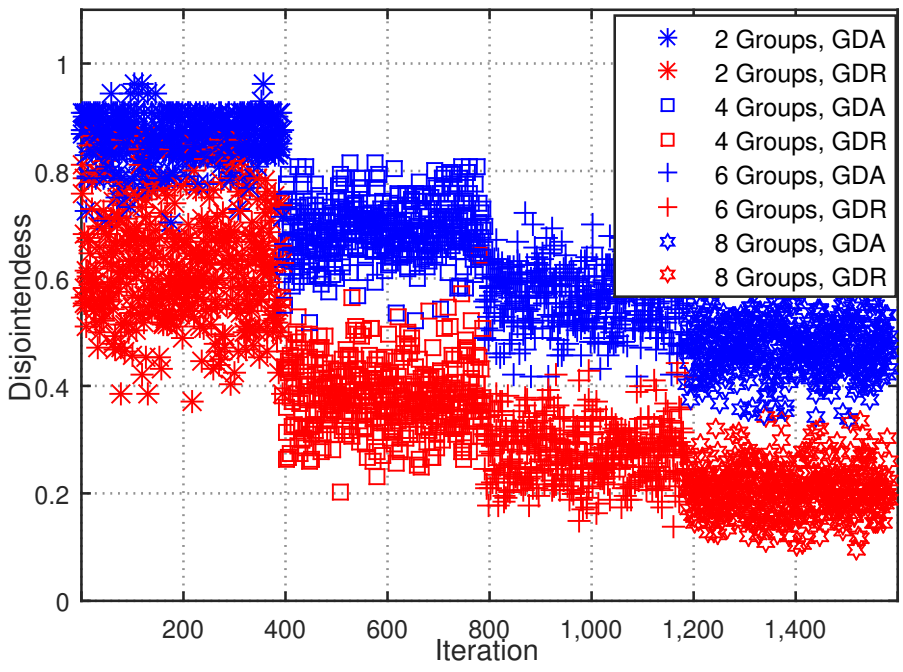

Fig. 7. Comparison of disjointedness among the groups formed in Flocklab in GDA and GDR.

sired number of groups having quite high Disjointedness in comparison to GDR. The produced groups can exploit fine-grained frequencies pretty well for simultaneous intragroup communication activities with very high reliability, and quite low latency and radio-on time.

\section{CONCLUSION}

In several recent works, fine-grained frequencies have been shown to exhibit a great potential to make much better utilization of the available bandwidth in licence free ISM bands. However, to the best of our knowledge, the current work is the first one that ties up synchronous transmission with fine-grain frequencies in a very systematic way to maximize the in-parallel communication capability. Through a very extensive local experiments we show that time-aligned inter-group and intra-group communication activities can take the advantage of the well-known physical layer phenomena called capture-effect even in the interfrequency context. By the virtue of this, under synchronous transmission even a CFD of $1 \mathrm{MHz}$ and $2 \mathrm{MHz}$ can be exploited very successfully. Through extensive experiments in real testbed infrastructures, we have shown that using the proposed strategy, even a very small frequency band of width $2 \mathrm{MHz}$ to $5 \mathrm{MHz}$, can be used to carry out networkwide in-parallel intra-group dissemination very fast and reliably for a pretty large number of groups.

In order to resolve the scalability issue, application of divide-and-conquer based strategies in network is quite inevitable. In such a strategy, the network needs to get fragmented into pieces (groups) and the target protocol is supposed to run in parallel in each of these groups. Our proposed group formation strategy can directly be useful for such applications. Moreover, random allocation of frequencies from a given range results in blind assignment of the frequencies without caring about the spatial relationship among the groups. Thus, careful allocation of the frequencies using the detailed understating reported in this work can exploit the available frequencies much better. 
We plan for taking up these issues as our part of our future endeavors in this direction.

\section{REFERENCES}

[1] Vikas Hassija, Vinay Chamola, Vikas Saxena, Divyansh Jain Pranav Goyal, and Biplab Sikdar. A survey on iot security: application areas, security threats, and solution architectures. 2019 IEEE Access.

[2] Xiaoyu Ji, Yuan He, Jiliang Wang, Kaishun Wu, Ke Yi, and Yunhao Liu. Voice over the dins: improving wireless channel utilization with collision tolerance. In 2013 21st IEEE ICNP.

[3] Mobashir Mohammad, Manjunath Doddavenkatappa, and Mun Choon Chan. Improving performance of synchronous transmission-based protocols using capture effect over multichannels. In 2017 ACM TOSN.

[4] Yang Yu and Viktor K Prasanna. Energy-balanced task allocation for collaborative processing in networked embedded systems. In Proceedings of the 2003 ACM SIGPLAN.

[5] Jinghua Zhu, Jianzhong Li, and Hong Gao. Tasks allocation for real-time applications in heterogeneous sensor networks for energy minimization. In Eighth ACIS IEEE SNPD 2007.

[6] Jagnyashini Debadarshini, Chandra Shekhar, and Sudipta Saha. Fine-grained frequencies for simultaneous intra-group one-to-all dissemination. In 2020 17th IEEE MASS.

[7] Wilson So, Jean Walrand, Jeonghoon Mo, et al. Mcmac: A parallel rendezvous multi-channel mac protocol. In 2007 IEEE Wireless Communications and Networking Conference.

[8] Milica D Jovanovic and Goran Lj Djordjevic. Tfmac: Multi-channel mac protocol for wireless sensor networks. In 8th 2007 IEEE International Conference on Telecommunications in Modern Satellite, Cable and Broadcasting Services.

[9] Gang Zhou, Chengdu Huang, Ting Yan, Tian He, John A Stankovic, Tarek F Abdelzaher, et al. Mmsn: Multi-frequency media access control for wireless sensor networks. In IEEE INFOCOM 2006.

[10] Yafeng Wu, John A Stankovic, Tian He, and Shan Lin. Realistic and efficient multi-channel communications in wireless sensor networks. In 27th IEEE INFOCOM 2008.

[11] Feng Li, Jun Luo, Gaotao Shi, and Ying He. FAVOR: frequency allocation for versatile occupancy of spectrum in wireless sensor networks. In Proceedings of ACM Mobihoc, 2013.

[12] X. Xu, J. Luo, and Q. Zhang. Design of non-orthogonal multichannel sensor networks. In Proceedings of IEEE ICDCS, 2010.

[13] Federico Ferrari, Marco Zimmerling, Lothar Thiele, and Olga Saukh. Efficient network flooding and time synchronization with glossy. In Proceedings of ACM/IEEE IPSN, 2011.

[14] Olaf Landsiedel, Federico Ferrari, and Marco Zimmerling. Chaos: Versatile and efficient all-to-all data sharing and in-network processing at scale. In Proceedings of ACM SenSys, 2013.

[15] Federico Ferrari, Marco Zimmerling, Luca Mottola, and Lothar Thiele. Low-power wireless bus. In Proceedings of ACM SenSys, 2012.

[16] Yafeng Wu, John A Stankovic, Tian He, and Shan Lin. Realistic and efficient multi-channel communications in wireless sensor networks. In Proceedings of IEEE INFOCOM, 2008.

[17] Jingbin Zhang, Gang Zhou, Chengdu Huang, Sang Hyuk Son, and John A Stankovic. TMMAC: An energy efficient multi-channel mac protocol for ad hoc networks. In Proceedings of IEEE ICC, 2007.

[18] A. A. Bertossi, C. M. Pinotti, and R. B. Tan. Channel assignment with separation for interference avoidance in wireless networks. In IEEE TPDS, 14(3), 2003.

[19] G. EkbataniFard and R. Monsefi. MAMAC: A multi-channel asynchronous mac protocol for wireless sensor networks. In Proceedings of IEEE BWCCA, 2011.

[20] Youngmin Kim, Hyojeong Shin, and Hojung Cha. Y-MAC: An energy-efficient multi-channel mac protocol for dense wireless sensor networks. In Proceedings of IEEE IPSN, 2008.

[21] Jiwoong Lee, Jeonghoon Mo, Tran Minh Trung, Jean Walrand, and Hoi-Sheung Wilson So. Design and analysis of a cooperative multichannel mac protocol for heterogeneous networks. 2010 IEEE Transactions on Vehicular Technology.

[22] Manjunath Doddavenkatappa, Mun Choon Chan, and Ben Leong. Improving link quality by exploiting channel diversity in wireless sensor networks. In Proceedings of IEEE RTSS, 2011.
[23] Beshr Al Nahas, Simon Duquennoy, Venkatraman Iyer, and Thiemo Voigt. Low-power listening goes multi-channel. In Proceedings of IEEE DCOSS, 2014.

[24] Feng Li, Jun Luo, Gaotao Shi, and Ying He. ART: Adaptive frequency-temporal co-existing of zigbee and wifi. In 2016 IEEE Transactions on Mobile Computing.

[25] Chandra Shekhar and Sudipta Saha. Fine-grained frequencies to combat cross technology interference in iot: A measurement study. In 2020 IEEE ANTS.

[26] J. Jun, S. Yeon, T. Kundu, D. P. Agrawal, and J. Jeong. CACA: Link-based channel allocation exploiting capture effect for channel reuse in wireless sensor networks. In Proceedings of IEEE ICDCS, 2016.

[27] Michael Baddeley, Usman Raza, Aleksandar Stanoev, George Oikonomou, Reza Nejabati, Mahesh Sooriyabandara, and Dimitra Simeonidou. Atomic-sdn: Is synchronous flooding the solution to software-defined networking in iot? 2019 IEEE Access.

[28] Chun-Hao Liao, Yuki Katsumata, Makoto Suzuki, and Hiroyuki Morikawa. Revisiting the so-called constructive interference in concurrent transmission. In 2016 41st IEEE LCN.

[29] Georg von Zengen, Alexander Willecke, and Lars C Wolf. Investigating concurrent transmission using software defined radios. In EWSN 2018.

[30] F. Li, J. Luo, G. Shi, and Y. He. ART: Adaptive frequency-temporal co-existing of zigbee and wifi. In 2017 IEEE Transactions on Mobile Computing.

[31] Wendi Rabiner Heinzelman, Anantha Chandrakasan, and Hari Balakrishnan. Energy-efficient communication protocol for wireless microsensor networks. In Proceedings of the 33rd IEEE annual Hawaii international conference on system sciences.

[32] Paramasiven Appavoo, Ebram Kamal William, Mun Choon Chan, and Mobashir Mohammad. Indriya2: A heterogeneous wireless sensor network (wsn) testbed. In Proceedings of Springer TridentCom, 2018.

[33] Roman Lim, Federico Ferrari, Marco Zimmerling, Christoph Walser, Philipp Sommer, and Jan Beutel. Flocklab: A testbed for distributed, synchronized tracing and profiling of wireless embedded systems. In Proceedings of IEEE IPSN, 2013.

[34] Markus Schuß, Carlo Alberto Boano, Manuel Weber, and Kay Römer. A competition to push the dependability of low-power wireless protocols to the edge. In Proceedings of ACM EWSN, 2017.

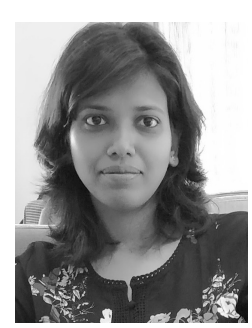

Jagnyashini Debadarshini received her Bachelors degree in Computer Science and engineering from the National Institute of Technology, Rourkela in 2011, and the Masters degree in Computer Science and engineering from Indian Institute of Technology, Bhubaneswar, in 2020. She is currently pursing her PhD at Indian Institute of Technology, Bhubaneswar. Her research interests are mainly in Internet-of-Things (IOT) and Wireless Sensor Networks.

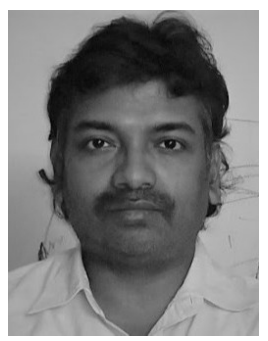

Sudipta Saha received his Masters and $\mathrm{PhD}$ degree in Computer Science and Engineering from Indian Institute of Technology, Kharagpur. $\mathrm{He}$ was associated with Singapore University of Technology and Design as well as National University of Singapore, as a Postdoc Research Fellow for several years. Currently, he is working as an Assistant Professor in the School of Electrical Sciences, in the discipline of Computer Science and Engineering, at Indian Institute of Technology Bhubaneswar, India. His primary research interest is in large scale decentralized systems, Wireless Sensor Networks, Internet-of-Things (IoT), Multi-Robot Systems, Secure Communication, and Complex Systems. 